\title{
Annie Jolivet, Anne-Françoise Molinié, Serge Volkoff (coord.), Le Travail avant la retraite. Emploi, travail et savoirs professionnels des seniors
}

Rueil-Malmaison, Éditions Liaisons, coll. « Collection Liaisons sociales », 2014

\section{Benoît Rapoport}

\section{OpenEdition}

Journals

Édition électronique

URL : http://journals.openedition.org/travailemploi/7075

DOI : 10.4000/travailemploi.7075

ISSN : 1775-416X

Éditeur

DARES - Ministère du Travail

\section{Édition imprimée}

Date de publication : 1 avril 2016

Pagination : 133-136

ISSN : 0224-4365

Référence électronique

Benoît Rapoport, « Annie Jolivet, Anne-Françoise Molinié, Serge Volkoff (coord.), Le Travail avant la retraite. Emploi, travail et savoirs professionnels des seniors », Travail et Emploi [En ligne], 146 | avril-juin 2016, mis en ligne le 11 juillet 2019, consulté le 24 septembre 2020. URL : http://

journals.openedition.org/travailemploi/7075; DOI : https://doi.org/10.4000/travailemploi.7075 


\title{
Le Travail avant la retraite. Emploi, travail et savoirs professionnels des seniors
}

\author{
Annie Jolivet, Anne-Françoise Molinié, Serge Volkoff (coord.)
}

Rueil-Malmaison, Éditions Liaisons, coll. « Collection Liaisons sociales », 2014, 225 p.

Lu par Benoît Rapoport

L'impact du vieillissement de la population ayant été insuffisamment anticipé en France, comme dans d'autres pays, les réformes des retraites se sont succédé depuis 1993 dans le but de rétablir l'équilibre du système par répartition. Les principaux instruments utilisés visent à inciter, voire à obliger, les personnes à reculer leur départ à la retraite. Les analyses des effets de ces mesures, ex post ou ex ante, ont porté essentiellement sur l'offre de travail et sur les conditions susceptibles d'agir sur les comportements des travailleurs. Elles se sont peu penchées sur les questions de la demande de travail et des conditions dans lesquelles ces départs plus tardifs allaient se dérouler. C'est ce manque que vient en partie combler Le Travail avant la retraite, ouvrage collectif coordonné par Annie Jolivet, Anne-Françoise Molinié et Serge Volkoff - par ailleurs pionniers de cette importante question -, en combinant différentes approches méthodologiques afin de cerner les conditions d'emploi des seniors.

L'ouvrage regroupe ainsi des contributions de démographes, d'économistes, d'ergonomes, de sociologues et de statisticiens pour la plupart liés au Centre d'études de l'emploi, et la variété des méthodes qui y sont utilisées concourt à sa richesse. Il s'articule autour de trois parties comptant trois chapitres chacune. La première porte sur l'emploi des seniors, la seconde sur les caractéristiques des emplois et les conditions de travail, et la troisième sur la transmission des savoirs, ce qui permet, en couvrant divers aspects du travail avant la retraite, d'éclairer le lecteur sur la place des seniors dans l'entreprise et son évolution.

Les politiques publiques en direction des seniors se sont longtemps contentées de chercher à gérer les flux de sortie de l'emploi des plus de 55 ans, avec, depuis 25 ans, l'objectif de les maintenir davantage en emploi. Le premier chapitre montre bien le caractère non linéaire de la succession des mesures adoptées, tout en rappelant les raisons ayant présidé à leur mise en place, particulièrement lorsqu'elles semblent contredire l'objectif du maintien en emploi (départs anticipés pour carrière longue par exemple). Certaines se traduisent dans les faits par le seul maintien en activité, donc

\footnotetext{
* Université Paris 1 ; Institut national d'études démographiques (Ined).
} 
souvent par du chômage. Les deux chapitres suivants s'intéressent à deux modes de gestion des flux : d'abord de sortie, en analysant l'utilisation de la rupture conventionnelle (chapitre 2), puis d'entrée, en interrogeant les pratiques de recrutement des seniors (chapitre 3). Ils ont le mérite de mettre en évidence que les seniors restent traités différemment des autres classes d'âge par leurs employeurs actuels ou potentiels. Les seniors semblent ainsi subir plus fortement que les plus jeunes la rupture conventionnelle qui devient de fait un outil de licenciement aux conséquences souvent funestes : bien que se déclarant majoritairement en recherche d'emploi à la suite de la rupture, les seniors retrouvent ensuite moins fréquemment un emploi. Les raisons peuvent en être trouvées dans les différences selon l'âge des pratiques de recrutement des entreprises. Ainsi, les caractéristiques des personnes recrutées et des entreprises les recrutant diffèrent fortement selon qu'il s'agit de seniors ou de candidats plus jeunes, avec plus de peu et de très qualifiés parmi les seniors, qui sont aussi moins souvent mis en concurrence. Deux études qualitatives montrent les différences entre secteurs, même si dans les deux cas les jugements négatifs sur les seniors sont souvent imputés aux clients par les employeurs. Les pratiques semblent plus discriminatoires à l'encontre des seniors dans le secteur informatique, tandis que, dans la grande distribution, ils sont jugés plus stables. Toutefois, alors qu'ils se déclarent satisfaits des seniors en emploi et qu'ils ont mis en place formations et allégement des contraintes à leur intention, les employeurs du secteur paraissent paradoxalement réticents à en embaucher de nouveaux. Si ces deux chapitres s'appuient sur des données originales - une enquête de la Dares dédiée à la rupture conventionnelle pour l'un et la combinaison des approches quantitative et qualitative pour l'autre -, l'absence de données de cadrage statistique laisse ouverte la question de la généralisation des résultats : que représente quantitativement le dispositif de rupture conventionnelle, et qu'en déduire sur l'ensemble des flux de sortie d'emploi ? Quelles sont les caractéristiques des populations étudiées au regard de l'ensemble des seniors, ou plus largement des personnes présentes sur le marché du travail ? Ainsi, l' analyse statistique du chapitre 3 ne dit rien des différences probables de parcours passé selon l'âge des candidats, tandis que le chapitre 2 ne va pas au-delà de la distinction par sexe et catégorie socioprofessionnelle : comment joue l'ancienneté par exemple?

Si les seniors paraissent traités différemment des autres classes d'âge en ce qui concerne les entrées et sorties de l'entreprise, leurs spécificités semblent à l'inverse insuffisamment prises en compte dans la gestion interne de la main-d'œuvre. Donc, même en cas de succès des politiques incitant à leur maintien en emploi, comment évaluer et améliorer les conditions dans lesquelles ils poursuivent leur activité ? C'est cette question fondamentale, peu présente dans le débat public, que pose la deuxième partie de l'ouvrage, avançant quelques premières propositions concrètes. L'analyse ergonomique du vieillissement au travail (chapitre 1) met ainsi en évidence l'évolution au cours des âges des interactions entre le travailleur et son environnement, en rappelant leur complexité et leur dépendance au contexte, le travailleur subissant son environnement tout en étant en mesure de le modifier. C'est de l'incapacité de saisir 
cette complexité que découlent en partie les stéréotypes liés à l'âge. La contribution conclut en plaidant à la fois pour une plus grande souplesse dans l'organisation du travail et la création de points d'ancrage limitant l'évolution accélérée des règles et des objectifs de travail afin de permettre l'accumulation de l'expérience. Sur un plan plus factuel, le deuxième chapitre, s' appuyant sur une enquête européenne, montre que l'exposition aux contraintes physiques ou temporelles reste élevée pour les seniors et décline plus tardivement pour les femmes que pour les hommes, certes initialement plus exposés. Le sentiment d'avoir moins de possibilités d'apprendre croît, quant à lui, dès 50 ans pour les deux sexes. L'ensemble de ces contraintes a pour conséquence que les femmes ont du mal à anticiper et à avoir la certitude qu'elles auront la capacité à exécuter le même travail après 60 ans, avec une forte hétérogénéité selon les pays. On peut reprocher à ce chapitre sa trop grande richesse, le format retenu ne permettant pas d'aller au bout de certaines questions : ainsi, la partie descriptive sur les contraintes ne distingue pas les pays. L'insuffisante prise en compte moyenne des spécificités liées à l'âge peut de fait masquer la diversité des situations entre pays, secteurs, et même entreprises. Le chapitre 3 le montre, en analysant une sélection d'accords seniors. Les entreprises sont souvent allées au-delà de la simple recherche de l'amélioration des conditions de travail, passant d'actions initialement individualisées à des dispositifs plus collectifs, ou étendant leur champ d'action, par anticipation, aux jeunes travailleurs exposés à des facteurs de risque de pénibilité. Les auteurs insistent en outre sur le fait que l'efficacité de ces actions est étroitement liée à la prise en compte des questions de performance de l'entreprise.

Au-delà des objectifs de maintien des seniors en emploi, la question des raisons pour lesquelles les entreprises souhaiteraient les maintenir reste ouverte. La nécessité de transmettre les savoirs professionnels est un argument utilisé pour tenter de les convaincre de conserver leurs seniors en leur gardant un rôle central, tout en évitant de perdre des savoirs accumulés et en intégrant les plus jeunes. Mais sa pertinence ne va pas de soi dans un contexte d'évolution permanente et de risque d'obsolescence des savoirs. La troisième partie de l'ouvrage aborde cette question en mettant en évidence sa grande complexité, parfois ignorée. Le chapitre 1 fournit un exemple éclairant des multiples facettes du processus de transmission en s'appuyant sur des observations et des entretiens menés dans un centre hospitalier universitaire : il montre que les formalisations théoriques usuelles de la transmission comme propagation d'un signal d'un émetteur à un récepteur omettent souvent le fait que les connaissances évoluent en permanence, que l'expérience n'est pas nécessairement codifiable et que la transmission ne se fait pas seulement par observation ou instructions mais nécessite de créer une véritable relation entre les partenaires. Il en ressort notamment que sous contrainte temporelle forte, la transmission peut facilement concurrencer la production, ce qui impose de les articuler. Plus généralement, la transmission par les seniors, même informelle, est plus aisée et donc plus fréquente si leur environnement de travail n'a pas connu de changements technologiques ou organisationnels ou si, après un tel changement, des formations ont été dispensées. La rapidité des évolutions et le 
défaut de formation adéquate des seniors expliquent que les plus de 50 ans restent paradoxalement sous-représentés parmi les formateurs, même dans le contexte du contrat de génération (chapitre 2). Le dernier chapitre analyse certaines réticences aux changements des travailleurs âgés, qui, loin de se réduire à un prétendu « conservatisme », peuvent aussi provenir d'un souci de conserver la qualité des relations nouées avec les collègues ou les clients. Une série d'entretiens met ainsi en évidence l'inquiétude des salariés face aux changements des environnements de gestion, à la quantification croissante de la production et à l'accélération des rythmes de travail. Le remplacement de la communication verbale par le mail se traduit pour eux par un appauvrissement des relations humaines néfaste à la circulation de l'information, ce qui conduit certains à se recréer un rôle de « liant », pas toujours identifié comme tel par leur hiérarchie. On peut ici regretter que ne soit pas suffisamment discuté le discours des salariés qui voient les transformations comme une régression sans envisager que la relation humaine et son mode de gestion puissent se transformer en retour.

Cette dernière partie présente des éléments particulièrement originaux en montrant que les discours sur les savoirs et leur transmission ne s'accommodent pas facilement de la complexité des processus. Néanmoins, elle laisse de nombreuses questions ouvertes lorsque la spécificité des savoirs détenus par les seniors est moins évidente. Que faire en pratique en cas de forte obsolescence des savoirs ? Comment assurer une place aux seniors lorsqu'il est trop coûteux de les former et difficile de transformer leur emploi ? Comment faire accepter aux plus jeunes et aux entreprises que les plus âgés soient traités différemment s'ils n'ont pas de spécificités particulières ? Le dernier chapitre, en mettant en évidence l'importance des compétences relationnelles au-delà du savoir technique, apporte quelques réponses invitant à discuter plus globalement les évolutions actuelles du lien au travail.

On pourra regretter qu'à l'exception d'une seule, les contributions restent centrées sur la France. Or si la plupart des pays occidentaux ont des objectifs de maintien des seniors en emploi, tous n'ont pas la même histoire de ce point de vue et certains ont pu créer des conditions favorables à ce maintien en emploi, par exemple en développant le temps partiel choisi. On aurait souhaité une conclusion qui, reprenant des recommandations à l'usage des décideurs publics, aurait bénéficié de l'analyse d'expériences internationales. Elle aurait aussi permis d'interroger la généralisation des résultats nécessaire à la calibration de l'action publique. En soulignant l'importance de traiter sérieusement la complexité de la question de l'emploi à l'approche de la retraite et en montrant que les mécanismes à l'œuvre sont souvent subtils, l'objectif affiché de plaider pour une « approche élargie de l'emploi des seniors » est toutefois clairement atteint. 